\title{
Brightness selectivity in the motion aftereffect*
}

\author{
RAY OVER $\dagger$ and JACK BROERSE \\ University of Queensland, St. Lucia, Australia 4067
}

\begin{abstract}
Eighteen Ss were required to track the apparent motion of a stationary grating viewed af ter prolonged inspection of a moving grating. Measures were obtained with the inspection and test gratings identical in contrast but different in space-average luminance, or with luminance held constant and contrast varied. The aftereffect was reduced as the gratings differed in space-average luminance, but contrast exerted less uniform influence as a variable. Brightness-selectivity in the motion aftereffect is interpreted within the selective adaptation model of aftereffects as evidence that some detectors in human vision are conjointly tuned to space-average luminance and image motion.
\end{abstract}

A stationary pattern is seen as moving following prolonged exposure to contours undergoing linear, rotational, or radial motion. In each case, the aftereffect is in the opposite direction to image motion during inspection. The motion aftereffect is color-selective in that exposure to moving contours in red light generates a larger distortion when the stationary test pattern is seen in red than when it is seen in green light (Favreau, Emerson, \& Corballis, 1972; Lovegrove, Over, \& Broerse, 1972; Mayhew \& Anstis, 1972). These data have been nterpreted as indicating that at least some edge detectors in human vision are conjointly responsive to wavelength and the direction of image motion. The argument is that a shift in color from the inspection to test stage reduces the aftereffect to the extent that patterns in different colored light are signaled by different populations of neural detectors. It is assumed that a stationary figure in red light is normally represented by the relative activity of red/upwards and red/downwards detectors. Prior exposure to lines moving downward in red light adapts red/downwards detectors, and the subsequent imbalance in neural response when the stationary red test pattern is shown represents the figure as moving upwards. When the test pattern is viewed in green light, its properties are signaled by the relative activity of green/upwards and green/downwards detectors, neither of which are adapted by inspection in red light.

The reduction rather than total loss in motion aftereffect, where there has been a color shift from inspection to test, suggests either that color-motion detectors are broadly tuned to wavelength or that there also exist motion detectors that are nonselective to wavelength. The question of present interest is whether or not achromatic motion detectors are tuned to luminance. Two luminance parameters are relevant when the visual display is patterned-space-average luminance

\footnotetext{
*This research was supported by an award from the Australian Research Grants Committee.

$\nmid$ Requests for reprints should be sent to Ray Over, Department of Psychology, University of Queensland, St. Lucia 4067, A ustralia.
}

and luminance contrast [defined as $\mathrm{L}(\max )$ $\mathrm{L}(\min ) / \mathrm{L}(\max )+\mathrm{L}(\min )$, where $\mathrm{L}(\max )$ is the luminance of the brightest part of the display and L(min), the luminance of the least bright part] .

Mayhew and Anstis (1972) provided evidence suggesting that there were limited linkages between image motion and space-average luminance under conditions where contrast is held constant. They required Ss to view, for alternate periods, a spiral undergoing clockwise motion in light of medium instensity and counterclockwise motion in dim light. Luminance-contingent motion aftereffects resulted, subsequent to inspection, in a stationary spiral's being perceived as being in counterclockwise motion when viewed in light of medium intensity and in clockwise motion when seen in dim light. Adaptation to bright-clockwise and dim-counterclockwise combinations produced motion aftereffects that were partly contingent on luminance, in that the stationary test spiral was perceived as being in counterclockwise motion in bright light and did not seem to be moving when seen in light of medium intensity.

Mayhew and Anstis (1972) did not consider possible linkages between image motion and luminance contrast. However, Parker (1972) measured the tilt aftereffect, which has been attributed to selective adaptation of neural detectors in the same way as the motion aftereffect (see Barlow \& Hill, 1963a, b; Coltheart, 1971; Over, 1971), with luminance contrast within the display varied between inspection and test stages while space-average luminance was held constant. The aftereffect increased when the shift was from high to low contrast. Such data suggest that there may be detectors in human vision that are conjointly tuned to luminance contrast and the spatial attributes of edges.

The present experiment considers whether the aftereffect of linear motion is selective to space-average luminance and luminance contrast. Os were required to track the apparent motion of a stationary vertical grating following inspection of a vertical grating moving left to right. Measures were obtained with the inspection and test gratings identical in space-average luminance, but differing in contrast or identical in contrast but differing in luminance. 


\section{METHOD}

The aftereffect was measured by requiring $S$ to track the apparent motion of a stationary test grating presented after inspection of a grating moving left to right at $1 \mathrm{deg} / \mathrm{sec}$. Tracking was accomplished by movement of pen (located out of S's sight) at the apparent velocity of the test grating. The aftereffect was measured in terms of the tracing left by the pen on paper moving at $.25 \mathrm{~cm} / \mathrm{sec}$ orthogonally to the carriage, and the value used for data analysis was the amount of pen displacement in the 15-sec postinspection period. This index has been used in other studies of the motion aftereffect (e.g., Day \& Strelow, 1971; Over, Broerse, Crassini, \& Lovegrove, 1973), and it reflects the cumulative aftereffect over the test period.

The inspection and test stimuli were square-wave gratings ( 1 cycle/deg) generated on separate oscilloscope faces (Advance Model 1000S), using a technique similar to that described by Campbell and Green (1965). An external oscillator was used to deliver a $100-\mathrm{KHz}$ sine wave to the unit's $\mathrm{Y}$-axis, and the signal was strengthened by the unit's internal amplifier to produce a raster. The horizontal $X$-axis was driven by the internal time-base circuit, and the electron beam was modulated to produce a square-wave grating by connecting a variable square-wave generator to the beam luminance control of the $\mathrm{Z}$-axis. Variation in the frequency of this modulating square wave had the effect of varying both the spatial frequency of the pattern on the CRO face and the velocity and direction of movement across the face. Periodicity and motion values reflected the difference in frequency between the square wave modulating the beam luminance and the scan frequency of the internal time base circuit. A stationary pattern resulted when one frequency was an integral harmonic of the other, and it was also possible to arrest the pattern at any intermediate off-harmonic point by gating the square wave through the external trigger socket of the oscilloscope.

The velocity of the moving grating was monitored during the adaptation period by converting the time-base waveform to a square pulse train. Each pulse coincided with the retrace edge, yielding a constant reference puls that was connected through a NAND gate together with the modulating square wave. An output pulse resulted when the reference pulse coincided with the high portion of the modulating square wave (Logical State 1), and a number of such coincidental states produced a block of pulses that were subsequently integrated to give a single high, monitored by a digital counter (Advance Model TC7) in conjunction with a laboratory interval generator. The period of the integrated output waveform was thus coincident with the passage of one vertical bar past the retrace edge of the time-base sawtooth, which served as the constant reference point. The interval generator gave sampling periods of $1 \mathrm{sec}$ or more, resulting in a recurrent readout of the number of bar passes per second.

An optical system allowed the inspection and test gratings to be viewed in succession in the same location within the visual field. Each grating was seen through a circular aperture subtending $3 \mathrm{deg} 20 \mathrm{~min}$ at $100 \mathrm{~cm}$. Because Day and Strelow (1971) found that the af tereffect of linear motion cannot be induced unless there is an illuminated background, the off-white surround to the aperture was maintained at $8.0 \mathrm{~cd} / \mathrm{m}^{2}$. The testing room was otherwise dark.

In one stage of the experiment, the space-average luminance of the inspection and test gratings was kept constant at $15.4 \mathrm{~cd} / \mathrm{m}^{2}$, and there were three contrast levels $(.03, .43$, and 91). The aftereffect was measured under nine conditions involving the three levels of contrast during inspection paired with each of the three levels in the test stage. The expectation is that if the aftereffect is contrast-specific, there will be a reduction in perceived distortion when contrast is changed between the inspection and test presentations. The aftereffect was similarly measured, with the contrast of the inspection and test gratings kept at .23 and the space-average luminance (1.4, 21.6 , and $\left.45.3 \mathrm{~cd} / \mathrm{m}^{2}\right)$ varied factorially between inspection and test stages. The question of interest was whether the motion aftereffect was larger when the inspection and test patterns were identical in space-average luminance or when they differed. The 18 undergraduate students who served as Ss were each tested under the nine Iuminance and the nine contrast combinations of inspection and test values, and the sequence in which conditions were tested was varied across Ss by a Latin square design. Under each condition, $S$ initially viewed the inspection grating for $3 \mathrm{~min}$ before a 15 -sec test measure was obtained. An additional $90-\mathrm{sec}$ inspection was given before another test setting was made. Testing continued under a further condition after a rest period of $6 \mathrm{~min}$.

\section{RESULTS}

The size of the motion aftereffect was given by the amount of pen displacement over a $15-\mathrm{sec}$ test probe. Mean displacement values (in millimeters) are shown separately in Fig. 1 for the contrast and luminance combinations of the inspection and test stimuli studied in the experiment. The test-retest reliability of settings under each condition was +.83 . Although there were inter-S differences in the overall extent of pen movement, conversion of raw scores to a common base (e.g., percentage of largest pen movement made by S) did not alter the overall trends found by analysis of variance. Mean absolute rather than relative measures are reported in Fig. 1 to allow comparison with other studies (e.g., Day \& Strelow, 1971; Lovegrove, Over, \& Broerse, 1972; Over, Broerse, Crassini, \& Lovegrove, 1973) in which the linear motion aftereffect has been expressed in terms of amount of pen displacement.

An analysis of variance was undertaken to establish whether the relative contrast and luminance of the inspection and test stimuli controlled the size of the aftereffect. The interaction between the level (low, medium, high) of the inspection and test stimuli was significant, $F(4,136)=6.58, p<.01$, but there was a significant three-way interaction between contrast vs luminance, inspection level, and test level, $F(4,136)=$ $4.62, p<.01$. Neither the main effects-contrast vs luminance, $F(1,34)=0.25, p>.05 ;$ level of the inspection stimulus, $F(2,68)=2.52, p>.05$; level of the test stimulus, $F(2,68)=0.26, p<.05)$ nor the remaining interactions were significant.

Comparisons by Duncan's new multiple range test showed that, with space-average luminance constant, variation in contrast between the inspection and test gratings exerted limited influence over the three-way interaction means. The combination low-high generated a significantly smaller aftereffect $(p<.05)$ than high-high, but the direction and extent of the contrast shift was unimportant at other levels of the test stimulus. There was no difference in mean aftereffect between low-low, medium-medium, and high-high combinations, indicating that absolute contrast was not effective.

The motion aftereffect was also insensitive to absolute 
Fig. 1. Mean aftereffect as a function of the contrast and space-average luminance of the test grating, with the inspection grating at low $(0)$, medium $(\Delta)$, and high (a) contrast and at low $(\bullet)$, medium $(\bullet)$, and high (a) space-average luminance.
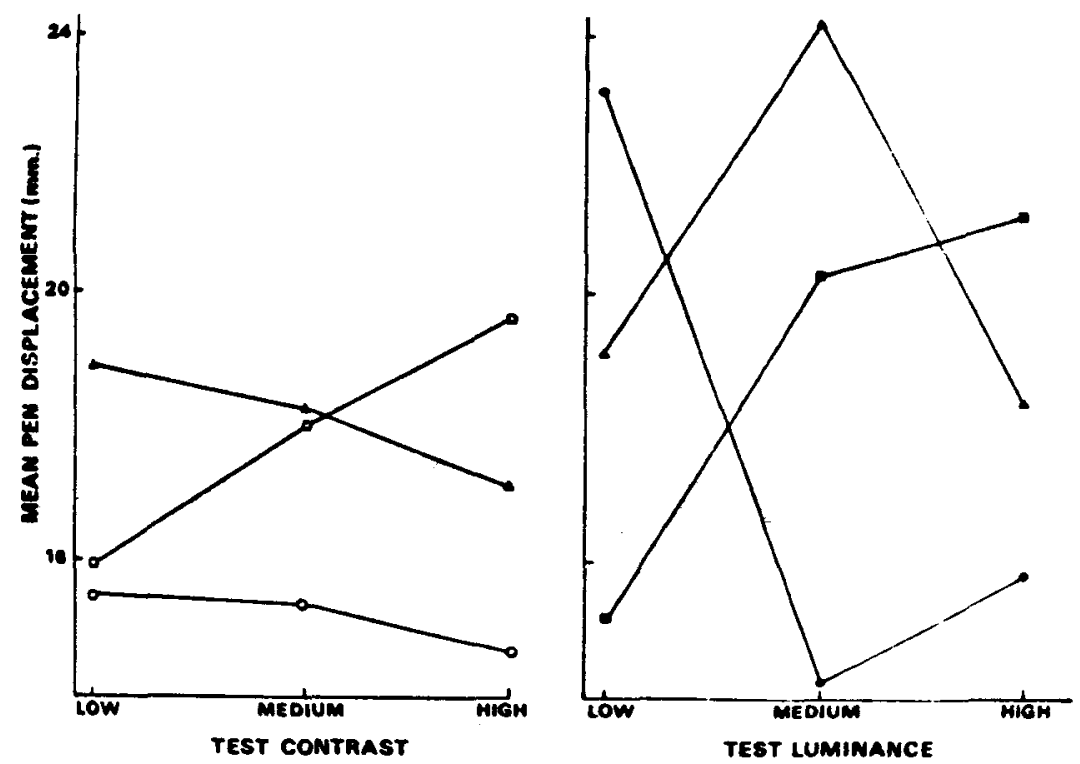

space-average luminance when contrast was unchanged, in that the luminance combinations of low-low, medium-medium, and high-high generated approximately the same amount of aftereffect. Relative luminance was, however, an effective variable, with a shift in luminance from inspection to test modifying the aftereffect in a systematic manner. The mean aftereffect was significantly larger for high-high than for low-high (but not medium-high), for medium-medium than for low-medium (but not high-medium), and for low-low than for medium-low or high-low.

\section{DISCUSSION}

The results show that the size of the motion aftereffect obtained when a stationary grating is viewed following inspection of a moving grating is independent of the absolute (low-low, medium-medium, high-high) contrast or space-average luminance of the gratings. In general, a shift in contrast between inspection and test presentations does not modify the size of the aftereffect. However, relative space-average luminance was effective as a variable in that the aftereffect was maximal when luminance was unchanged from inspection to test, and in general was reduced when luminance was increased or decreased.

The present results can be contrasted with the incomplete luminance-selectivity demonstrated by Mayhew and Anstis (1972) for the spiral aftereffect. Mayhew and Anstis required Ss to inspect the spiral undergoing clockwise motion at one luminance level and counterclockwise motion at another luminance level. The Ss were subsequently asked to report the apparent direction of motion of a stationary spiral viewed at each luminance level. Motion aftereffects were selective to luminance when the opposite directions of motion during inspection had been viewed in dim and medium light, but, following inspection of clockwise motion in bright light and counterclockwise motion in medium light, the stationary spiral was perceived as being in counterclockwise motion in bright light but no aftereffect was obtained in medium light. relative strengths of opposed directional aftereffects, and on these grounds they would not provide a sensitive index of luminance selectivity in the motion aftereffect. The issue can be considered with reference to the present results, indicating that a unidirectional aftereffect induced at a specific luminance level generalizes across the luminance range. In these terms, exposure to a spiral in clockwise motion in bright light would result in a counterclockwise aftereffect being found later in medium as well as in bright light, and exposure to counterclockwise motion in medium light would generate a clockwise aftereffect in bright as well as in medium light. The test figure would appear stationary in medium light if the medium-clockwise and medium-counterclockwise aftereffects were equal in strength, but it would appear to be undergoing counterclockwise motion in bright light if the counterclockwise-bright aftereffect were stronger than the clockwise-bright aftereffect. The suggestionthat is being made is that the slopes of the generalization functions for the opposite directions of aftereffect determine whether partial or complete selectivity will be found using the method employed by Mayhew and Anstis (1972). This same factor is possibly at the basis of the transposition in luminance and color selectivity reported by Mayhew and Anstis. In the present experiment, the magnitude of the unidirectional motion aftereffect was measured as a function of the luminance level at inspection and test stages, and there was no need to consider the relative strengths of opposed directional aftereffects. The results that were obtained provide a
Mayhew and Anstis's results may, however, reflect the 
clear indication that the motion aftereffect is contingent on luminance.

There have been proposals (e.g., Blakemore \& Nachmias, 1971; Over, 1971) that contour masking, as well as spatial aftereffect paradigms, provide information on the selectivity of detectors mediating perception. Pantle and Sekuler (1969) have reported masking data that suggest that motion detectors are partly tuned to contrast. They measured the threshold for detection of a moving target after exposure to a moving adaptation grating exerted control over the extent of masking, but this applied only within the contrast range of .00 to .16 . Pantle and Sekuler's data are consistent with electrophysiological evidence (Barlow \& Hill, 1963) that directionally selective cells in the rabbit retina become more active as contrast increases up to .20 , but not beyond. An explanation of the aftereffect in terms of selective adaptation of contrast-specific motion detectors would therefore suggest that changes from low to high contrast or from high to low contrast should decrease the motion aftereffect, whereas changes from medium to high contrast or from high to medium contrast should be ineffective. In the present experiment, the change from low to high contrast did, in fact, produce a smaller aftereffect than when the contrast was high during inspection and test, but the aftereffect obtained with the transition from high to low contrast was of the same magnitude as that found when the contrast was low during inspection and test.

The selective adaptation model outlined earlier in relation to color-specific motion aftereffects can be extended to incorporate the present data on luminance selectivity. The necessary assumption is that some detectors in human vision respond to both the direction of image motion and the space-average luminance of patterned displays. The model supposes that detectors excited during inspection are suppressed afterwards, and that an aftereffect occurs through imbalance in the pattern of activity by which the test stimulus is normally signaled. In these terms, a stationary pattern in bright light is represented by the activity of bright/left-to-right and bright/right-to-left detectors, and an aftereffect is produced to the extent that one of these populations is adapted. The reduction, rather than total disappearance, in motion aftereffect when there was a large shift in space-average luminance from inspectionto test indicates either that luminance-motion detectors are broadly tuned to luminance or that there also exist motion detectors that are nonselective to space-average luminance.

It should be noted that the present proposals, that spatial detectors are tuned to space-average luminance rather than to contrast, receive little support from electrophysiological studies. Campbell and Kulikowski (1972) have demonstrated that the evoked potential found when human Ss view a grating is selective to luminance contrast when space-average luminance is held constant. This result is consistent with microelectrode evidence (e.g., Burns \& Pritchard, 1964) that spatially selective cells are sensitive to contrast. Henry and Bishop (1971) studied the response of cortical cells tuned to contour orientation and motion when contrast was unchanged and space-average luminance varied. Neural response was relatively unaffected by a shift of $2 \log$ units in luminance.

Brightness-spatial linkages in human vision can be further studied by selective adaptation paradigms. Mayhew and Anstis (1972) have noted, for example, that a vertical grating appears brighter than a horizontal grating of the same space-average luminance following alternate exposure to dim-vertical and bright-horizontal gratings. The implication is that some tilt detectors are also tuned to luminance in a manner analogous to the tilt-color pairings that are believed to underlie tilt-specific color aftereffects (McCollough, 1965). There would be interest in establishing whether spatial periodicity and space-average luminance are linked in view of demonstrations (Breitmeyer \& Cooper, 1972; Harris, 1970; Lovegrove \& Over, 1972; Stromeyer, 1972) that alternate exposure to a fine grating in red light and a coarse grating in green light results in the fine grating's appearing green and the coarse grating's appearing red when they are later seen in white light.

\section{REFERENCES}

Barlow, H. B., \& Hill, R. M. Evidence for a physiological explanation of the waterfall phenomenon and figural aftereffects. Nature, 1963a, 200, 1434-1435.

Barlow, H. B., \& Hill, R. M. Selective sensitivity to direction of movement in ganglion cells of the rabbit retina. Science. $1963 \mathrm{~b}, 139,412-414$.

Blakemore, C., \& Nachmias, J. The orientation specificity of two visual aftereffects. Journal of Physiology, 1971, 213, 157-174.

Breitmeyer, B. G., \& Cooper, L. A. Frequency-specific color adaptation in the human visual system. Perception \& Psychophysics, 1972, 11, 95-96.

Burns, B. D., \& Pritchard, R. Contrast discrimination by neurones in the cat's visual cerebral cortex. Journal of Physiology, 1964, 175, 445-463.

Campbell, F. W., \& Green, D. G. Optical and retinal factors affecting visual resolution. Journal of Physiology, London, $1965,181,576-593$.

Campbell, F. W., \& Kulikowski, J. J. The visual evoked potential as a function of contrast of a grating pattern. Journal of Physiology, 1972, 222, 345-356.

Coltheart, M. Visual feature-analyzers and aftereffects. Psychological Review, 1971, 78, 114-121.

Day, R. H., \& Strelow, E. Visual aftereffect of movement: Partial or complete reduction in the absence of a patterned surround. Nature, 1971, $230,55$.

Favreau, O., Emerson, V., \& Corballis, M. C. Movement aftereffects contingent on color. Science, 1972, 176, 78-79.

Harris, C. S. Effect of viewing distance on a color aftereffect specific to spatial frequency. Psychonomic Science, 1970, 21. 350.

Henry, G. H., \& Bishop, P. O. Simple cells of the striate cortex. In W. D. Neff (Ed.), Contributions to sensory physiolog: Vol. 5. New York: Academic Press, 1971

Lovegrove, W. J., \& Over. R. Color adaptation of spatial frequency detectors in human vision. Science, 1972, 176. $541-543$. 
Lovegrove, W. J., Over. R., \& Broerse, J. Color-selectivity of the motion aftereffect. Nature, 1972, 238, 334-335.

Mayhew, J. E. W., \& Anstis, S. M. Movement of aftereffects contingent on color, intensity, and pattern. Perception \& Psychophysics, 1972, 12, 77-85.

McCollough, C. Color adaptation of edge-detectors in the human visual system. Science, 1965, 149, 1115-1116.

Over. R. Comparison of normalization theory and neural enhancement explanation of negative aftereffects. Psychological Bulletin, 1971, 75, 225-243.

Over, R., Broerse, J., Crassini, B., \& Lovegrove, W. Spatial determinants of the aftereffect of seen motion. Vision Research, 1973, 13, in press.
Pantle, A., \& Sekuler, R. Contrast response of human visual mechanisms sensitive to orientation and direction of motion. Vision Research, 1969, 9, 397-406.

Parker, D. M. Contrast and size variables and the tilt aftereffect. Quarterly Journal of Experimental Psychology, 1972, 24, 1-7.

Stromeyer, C. F. Edge-contingent color after effects: Spatial frequency specificity. Vision Research, 1972, 12. 717-733.

(Received for publication November 7, 1972; final revision received July 5,1973 .) 